\title{
SOME PROPERTIES OF FUNCTIONS OF EXPONENTIAL TYPE
}

RICHARD DUFFIN AND A. C. SCHAEFFER

Suppose that $f(z)$ is an entire function such that

$$
f(z)=O\left(e^{\lambda|z|}\right),
$$

and on the real axis $f(z)$ is real and bounded by 1 . First it is shown that the function $\cos \lambda z-f(z)$ cannot have complex zeros. Moreover its real zeros are simple at the points where the strict inequality $|f(z)|<1$ is satisfied. This theorem is then used to find a "best possible" dominant over the complex plane of the class of functions $f(z)$. Finally it is shown that these results contain two theorems of S. Bernstein.

THEOREM 1. Let $f(z)$ be an entire function of $z=x+i y$, real for real $z$, and satisfying the conditions:

$$
|f(x)| \leqq 1
$$

on the real axis, and

$$
|f(z)|=O\left(e^{\lambda|z|}\right), \quad \lambda>0,
$$

uniformly over the entire plane. Then for every real $\alpha$ the function

$$
\cos (\lambda z+\alpha)-f(z)
$$

has only real zeros, or vanishes identically. Moreover all the zeros are simple, except perhaps at points on the real axis where $f(x)= \pm 1$.

In the proof of Theorem 1 we shall use the following result of Pólya and Szegö,* which we state as a lemma.

LEMma 1. If $f(z)$ satisfies the conditions of Theorem 1 , then actually

$$
|f(z)| \leqq e^{\lambda|v|} \text {. }
$$

Proof. By the hypotheses of Theorem 1 the function $f(z) e^{i \lambda z}$ is bounded on the positive halves of the real and imaginary axes and is $O\left(e^{\lambda|z|}\right)$ in the angular region between them. Then by the PhragménLindelöf $\dagger$ principle $f(z) e^{i \lambda z}$ is bounded throughout the first quadrant. In the same way one shows that it is bounded in the second quadrant.

* G. Polya and G. Szegö, Aufgaben und Lehrsätze aus der Analysis, vol. 2, p. 36, prove this by a different method.

$\dagger$ E. C. Titchmarsh, Theory of Functions, p. 177. 
Then $f(z) e^{i \lambda z}$ is bounded by 1 on the real axis and by some constant in the upper half-plane. Again applying the Phragmén-Lindelöf principle, this time with the angular region the upper half-plane, we see that $\left|f(z) e^{i \lambda z}\right| \leqq 1$ in the upper half-plane, so (3) is true for $y \geqq 0$. When we use the function $f(z) e^{-i \lambda z}$ the same method shows that (3) is true for $y \leqq 0$.

To prove Theorem 1 it will be sufficient to consider the function $\cos \lambda z-f(z)$, that is, the case $\alpha=0$. Let $\epsilon$ be some positive number less than 1 , and consider the function

$$
f_{\epsilon}(z)=\frac{\sin \lambda \epsilon z}{\lambda \epsilon z}(1-\epsilon) f((1-\epsilon) z) .
$$

By Lemma 1,

$$
\left|f_{\epsilon}(z)\right| \leqq \frac{e^{\lambda \epsilon|y|}}{\lambda \epsilon|z|}(1-\epsilon) e^{\lambda|y|(1-\epsilon)}<\frac{e^{\lambda|y|}}{\lambda \epsilon|z|} ;
$$

so if $y_{0}$ is sufficiently large, we have, on the lines $y= \pm y_{0}$,

$$
\left|f_{\epsilon}(z)\right|<|\cos \lambda z| \text {. }
$$

It follows from (4) that, if $K$ is a sufficiently large positive integer,

$$
\left|f_{\epsilon}(z)\right|<|\cos \lambda z|
$$

on the lines $x= \pm K \pi / \lambda$.

Let $\zeta$ be a closed rectangular contour consisting of segments of the lines $x= \pm K \pi / \lambda, y= \pm y_{0}$. We have shown that $|\cos \lambda z|>\left|f_{\epsilon}(z)\right|$ on $\zeta$, so by Rouché's theorem* the function

$$
\cos \lambda z-f_{\epsilon}(z)
$$

has the same number of zeros in $\zeta$ as $\cos \lambda z$, that is, $2 K$ zeros. On the real axis $\left|f_{\epsilon}(x)\right| \leqq 1-\epsilon$, so at the points $\nu \pi / \lambda,(\nu=0, \pm 1, \pm 2, \cdots)$, we have $\left|f_{\epsilon}(z)\right|<|\cos \lambda z|$. Thus $\cos \lambda z-f_{\epsilon}(z)$ is alternately plus and minus at the $2 K+1$ points $\nu \pi / \lambda,(\nu=-K,-K+1, \cdots, K)$; so inside $\zeta$ it has at least $2 K$ real zeros. But we have shown that there are exactly $2 K$ zeros of $\cos \lambda z-f_{\epsilon}(z)$ in $\zeta$. Hence there are no complex zeros, and there is exactly one (simple) zero in each interval $(\nu \pi / \lambda,(\nu+1) \pi / \lambda),(\nu=-K, \cdots, K-1)$. Taking larger values of $y_{0}$ and $K$ we see that $\cos \lambda z-f_{\epsilon}(z)$ has exclusively real and simple zeros, which lie in the intervals $\nu \pi / \lambda<z<(\nu+1) \pi / \lambda$, ( $\nu$ integer, $-\infty<\nu<\infty)$.

When $\epsilon \rightarrow 0$ the function $\cos \lambda z-f_{\epsilon}(z)$ approaches $\cos \lambda z-f(z)$

* E. C. Titchmarsh, loc. cit., p. 116. 
uniformly in every bounded domain. But if the latter function is not identically zero it follows from a theorem of Hurwitz* that its zeros are limit points of the zeros of $\cos \lambda z-f_{\epsilon}(z)$. Thus $\cos \lambda z-f(z)$ cannot have non-real zeros; moreover it has an infinite number of real zeros which are all simple, except those at the points $\nu \pi / \lambda$ if $f(\nu \pi / \lambda)=(-1)^{\nu}$. Every interval $\nu \pi / \lambda<z<(\nu+1) \pi / \lambda$ at the endpoints of which $|f(z)|<1$ contains exactly one zero. If $f(\nu \pi / \lambda)=(-1)^{\nu}$, we have a double zero at $\nu \pi / \lambda$ but no further zeros in the interior or at the endpoints of the interval $((\nu-1) \pi / \lambda,(\nu+1) \pi / \lambda)$. This proves Theorem 1 .

We have shown that, if $f(z)$ satisfies the conditions of Theorem. 1 , then actually the inequality (3) is satisfied. There is, however, no such function for which (3) becomes an equality at points off the real axis. Using Theorem 1 we can show that the stronger inequality

$$
|f(z)| \leqq \cosh \lambda y,
$$

is satisfied. If $f(z) \equiv \cos (\lambda z+\alpha), \alpha$ real, then the equality holds along certain lines parallel to the imaginary axis.

THEOREM 2. If $f(z)$ satisfies the conditions of Theorem 1, then

$$
|f(z)| \leqq \cosh \lambda y
$$

and, unless $f(z)$ is of the form $\cos (\lambda z+\alpha)$, the equality can occur only on the real axis.

PROOF. It will be sufficient to show that (6) is true on the imaginary axis. Suppose $f(z)$ is not of the form $\cos (\lambda z+\alpha)$, and for some $y$, $(|y|>0)$, we have

$$
|f(i y)| \geqq \cosh \lambda y .
$$

From the expansion

$$
\cos (\lambda i y+\beta)=\cos \beta \cosh \lambda y-i \sin \beta \sinh \lambda y
$$

we see that $\cos (\lambda i y+\beta)$ has, for a suitable real $\beta$, the same amplitude as $f(i y)$. Since $|\cos (\lambda i y+\beta)| \leqq \cosh \lambda y \leqq|f(i y)|$, there is a real $\gamma$, $(0<\gamma \leqq 1)$, such that $|\gamma f(i y)|=|\cos (\lambda i y+\beta)|$. Then

$$
\cos (\lambda z+\beta)=\gamma f(z)
$$

at the point $z=i y$, since the amplitudes and magnitudes are the same. But $\gamma f(z)$ satisfies the conditions of Theorem 1, so $\cos (\lambda z+\beta)$ $-\gamma f(z)$ can have only real zeros. The contradiction proves Theorem 2.

* E. C. Titchmarsh, loc. cit., p. 119. 
It has been shown by S. Bernstein* that if $f(z)$ satisfies the conditions of Theorem 1 , then

$$
\left|f^{\prime}(z)\right| \leqq \lambda
$$

on the real axis. Szegö (for the case in which $f(z)$ is a trigonometric polynomial) and later Boas (under essentially the same conditions as in Theorem 1) obtained $\dagger$ the stronger inequality

$$
\left\{f^{\prime}(z)\right\}^{2}+\lambda^{2}\{f(z)\}^{2} \leqq \lambda^{2}
$$

on the real axis. The authors obtained a generalization of ( 7 ) for complex values of $z$ in a previous paper. Using Theorem 1 we can now prove this corollary:

CoRollary. If $f(z)$ satisfies the conditions of Theorem 1, then (7) is true on the real axis.

Proof. Suppose $f(z)$ satisfies the conditions of Theorem 1 and is not of the form $\cos (\lambda z+\alpha)$. At points of the real axis where $f(z)= \pm 1$ we must have $f^{\prime}(z)=0$, so $(7)$ is certainly true. Hence suppose that, at some point $z_{0},\left|f\left(z_{0}\right)\right|<1$, and (7) is not satisfied. Then by suitable choice of real $\gamma,(0<\gamma \leqq 1)$, we have the equality

$$
\left\{\gamma f^{\prime}(z)\right\}^{2}+\lambda^{2}\{\gamma f(z)\}^{2}=\lambda^{2} .
$$

Then since $\cos (\lambda z+\alpha)$ satisfies the differential equation

$$
\left[\frac{d}{d z} \cos (\lambda z+\alpha)\right]^{2}+\lambda^{2}[\cos (\lambda z+\alpha)]^{2}=\lambda^{2},
$$

there is a real $\alpha$ so that, at the point $z_{0}$,

$$
\cos (\lambda z+\alpha)=\gamma f(z), \quad \frac{d}{d z} \cos (\lambda z+\alpha)=\gamma f^{\prime}(z) .
$$

Thus the function

$$
\cos (\lambda z+\alpha)-\gamma f(z)
$$

has a double zero at the point $z_{0}$, where $\left|\gamma f\left(z_{0}\right)\right|<1$; but by Theorem 1 this is impossible. The contradiction proves the corollary.

Using Theorem 2 we prove a second theorem $\ddagger$ of S. Bernstein.

* S. Bernstein, Comptes Rendus, vol. 176 (1923), p. 1603.

$\dagger$ G. Szegö, Schriften der Königsberger Gelehrten Gesellschaft, Naturwissenschaftliche Klasse, vol. 5 (1928), p. 69; R. P. Boas, Transactions of this Society, vol. 40 (1936), p. 287. See also Van der Corput and Schaake, Compositio Mathematica, vol. 2 (1935), p. 321; R. J. Duffin and A. C. Schaeffer, this Bulletin, vol. 43 (1937), p. 554.

¥ S. Bernstein, Communications de la Société Mathématique de Charkow, (2), vol. 14. See also M. Riesz, Acta Mathematica, vol. 40 (1916), p. 337. 
BeRnSTEIN's THEOREM. Let $P(s)$ be a polynomial of degree $n$ or less with real coefficients such that in the interval $(-1,1)$ of the real axis $|P(s)| \leqq 1$. If $A$ and $B$ are the semi-axes of an ellipse passing through the point $s$ and having foci at the points 1 and -1 , then

$$
|P(s)| \leqq(A+B)^{n} \text {. }
$$

We shall prove that if $P(s)$ satisfies the conditions of Bernstein's theorem, then the stronger inequality

$$
|P(s)| \leqq \frac{1}{2}\left\{(A+B)^{n}+(A+B)^{-n}\right\}
$$

is satisfied.

Proof. If $P(s)$ satisfies the conditions of Bernstein's theorem it is clear that $P(\cos z)$ is a polynomial of degree $n$ in $\cos z$ and is bounded by 1 on the real axis, so it satisfies the conditions of Theorem 2 with $\lambda=n$. Then we have

$$
|P(\cos z)| \leqq \cosh n y,
$$

where $z=x+i y$. Let $s=\sigma+i t$ be any fixed point not in the interval $(-1,1)$ of the real axis, and choose a $z$ so that $s=\cos z$. Then we have the relations

$$
\begin{aligned}
\sigma & =\cos x \cosh y, \\
t & =-\sin x \sinh y,
\end{aligned}
$$

and on eliminating $x$ we obtain the equation

where

$$
\frac{\sigma^{2}}{A^{2}}+\frac{t^{2}}{B^{2}}=1
$$

$$
A=\cosh |y|, \quad B=\sinh |y| \text {. }
$$

Thus the point $s$ lies on an ellipse with center at the origin and semiaxes $A$ and $B$, and the foci are at the points 1 and -1 since $A^{2}-B^{2}=1$. Since $A+B=e^{|y|}$ we see that

$$
\cosh n y=\frac{1}{2}\left\{(A+B)^{n}+(A+B)^{-n}\right\},
$$

and on putting this in (11) we have (10), namely,

$$
|P(s)|=|P(\cos z)| \leqq \frac{1}{2}\left\{(A+B)^{n}+(A+B)^{-n}\right\} \text {. }
$$

This is a "best possible" inequality in the sense that if $P(s)$ is the $n$th Tchebycheff polynomial, $T_{n}(s)=\cos \left(n \cos ^{-1} s\right)$, then (10) becomes an equality along certain lines in the complex plane.

Purdue University 